\title{
BILATERAL PERIPAPILLARY CHOROIDAL NEOVASCULARIZATION ASSOCIATED WITH AGE-RELATED MACULAR DEGENERATION: A CASE REPORT
}

\author{
Dručka Eva*, Elksnis Ēriks*,**, Šepetiene Svetlana*,**, Ozolinš Artūrs*,** \\ * Riga Stradins University, Dzirciema iela 16, Riga, LV-1007, Latvia \\ * Pauls Stradiņš Clinical University, Pilsonu iela 1, Riga, LV-1002, Latvia
}

\section{Summary}

A 50-year-old female was diagnosed with significant peripapillary choroidal neovascularization (PCNV) in the left eye (LE). 12 months after a close follow-up, 4 intravitreal injections with bevacizumab and retinal laser photocoagulation were performed to limit the distribution of the lesion. A year after the primary diagnosis in the LE, a tiny PCNV also developed in the right eye (RE). Simultaneous activity was additionally observed in both peripapillary lesions at the time. At the last follow-up, after 43 months, the best corrected visual acuity in the RE was 0.8 , but in the LE it was only 0.2 . In total, the LE received 6 intravitreal injections with bevacizumab, while the $\mathrm{RE}$ received 3 injections.

The case report concludes that early diagnosis and intervention helps maintain visual acuity better in the cases of a small lesion than in developed significant PCNV with intense activity characterised with oedema, hard exudates and haemorrhages.

Keywords: Peripapillary choroidal neovascularization, age-related macular degeneration, anti-VEGF

\section{AIM OF THE DEMONSTRATION}

To reflect a rare clinical case in ophthalmology of bilateral peripapillary choroidal neovascularization (PCNV) with atypical presentation and applied treatment modalities to improve the outcome.

\section{CASE REPORT}

A 50-year-old female presented to a regional ophthalmologist for a regular once-a-year examination of the eyes. At that examination, no complaints regarding patient's vision were expressed. No significant pathologies had been reflected at the previous eye examination.

According to the general anamnesis, the patient had primary arterial hypertension well compensated with beta blockers and dyslipidaemia treated with atorvastatin. Sectoral resection of the left side breast cancer had been performed 3 years ago, followed by chemotherapy with tamoxifen and brachytheraphy. Regular controls had not revealed any metastasis.

Ophthalmic examination after decimal scale indicated that the best corrected visual acuity (BCVA) in the right eye (RE) was 1.0, while in the left eye (LE) it was 0.8. Comparing to the last year, there was a loss of 2 lines of visual acuity in the LE. Intraocular pressure in both eyes $(\mathrm{BE})$ was $16 \mathrm{mmHg}$.

Biomicroscopy results of anterior segment were insignificant for BE.

Fundoscopy of the RE reflected tiny changes in blood vessels and small drusen in the macula. The optic nerve disc was characterised by central excavation, preserved neuroretinal rim and peripapillary atrophy.
In contrast, the fundoscopic findings of the LE were considerably different. A prominent pink-coloured lesion directly above the optic nerve disc occupying 4 clock hours of the disc circumference was observed. The lesion was surrounded by hard exudates, oedema, intraretinal and subretinal haemorrhages (Fig. 1). The central region of macula was occupied by large soft drusen and hyperpigmentation.

Because of the previous anamnesis of breast cancer, there was a high suspicion of metastasis in choroidea. Further retinal examinations including optical coherence tomography (OCT) and fluorescein angiography (FA) were thus ordered.

OCT demonstrated a sub-retinal pigment epithelium (sub-RPE) hyperreflective lesion in the peripapillary area with a significant oedema in the LE. The FA revealed hypoflourescence in an early phase followed by lace-pattern hyperflourescence in an arterio-venous phase and leakage in a late phase (Fig. 2). According to the results of the examinations, a preliminary diagnosis of peripapillary choroidal neovascularization (PCNV) membrane type 1 in the LE was established. In the $\mathrm{BE}$ an age-related macular degeneration (AMD) was formulated.

Soon afterwards, the patient received 3 monthly intravitreal anti-vascular endothelial growth factor (anti-VEGF) injections with bevacizumab in the LE. This intervention led towards a rapid decrease of sub-RPE fluid. However, it promoted fibrovascular activity; thus, intense subretinal fibrosis developed in the region of the lesion. After 3 months, an activity of PCNV was noticed once again. In order to protect 
the macular central region from ingrown of new blood vessels and oedema, peri-lesion laser photocoagulation was performed, followed by a bevacizumab intravitreal injection.

The patient was under a close follow-up every month. After 12 months, a regular examination with OCT revealed new sub-RPE lesion now in the RE. The FA approved a tiny PCNV membrane in the RE. At the same time, PCNV in the LE indicated signs of activity. Therefore, the patient was prescribed to receive bevacizumab intravitreal injections in the BE.

At the last follow-up, after 43 months, the BCVA in the RE was 0.8 and 0.2 in the LE. In total, the patient received 3 intravitreal injections with bevacizumab in the RE and 6 in the LE. In the BE peripapillary lesion was inactive, while significant scaring was noticed in superior/temporal-superior part above the optic nerve disc in the LE, and choroidal neovascularization involved centre of fovea (Fig. 3).

\section{DISCUSSION}

According to its definition, choroidal neovascularization (CNV) includes abnormal growth of vessels from the choroidal vasculature to the neurosensory retina through the Bruch's membrane. Consequently, visual acuity is threated by retinal oedema, haemorrhages and neovascular membrane ingrown in macula. The progression of the disease disrupts retinal structure and function, leading towards irreversible vision loss (1). PCNV is a collection of new choroidal blood vessels within one disc diameter of the optic nerve head through Bruch's membrane or around the termination of the membrane adjacent to the disc. In most of these cases, patients do not experience any symptoms while the lesion is located outside the macula, like in the case of the present study (2).

Browning et al. described ocular conditions associated with PCNV as $45.2 \%$ age-related macular degeneration, $39.1 \%$ idiopathic, $4.3 \%$ multifocal choroiditis, $2.6 \%$ angioid streaks, $1.7 \%$, presumed ocular histoplasmosis, $1.7 \%$ choroidal osteoma, $0.9 \%$ optic disc drusen and $0.9 \%$ congenital disc anomalies (3). Furthermore, the natural course of untreated PCNV can vary regardless of the size and aetiology ranging from spontaneous involution to fulminant growing towards the fovea (2). PCNV is uncommon, accounting for less than $10 \%$ of all CNV (4). The Bridlington eye assessment project of PCNV identified population prevalence of $0.29 \%$, and $0.06 \%$ bilaterality in Caucasians (5).

PCNV is defined as large if it covers more than 3.5 disc areas or involves over $50 \%$ of the disc circumference (6). In the case of the present study, the LE PCNV met this criterion with extent around 4 disc areas. The findings in the RE could be defined as a small lesion. Larger PCNV possess a complication of causing visual decrease by inducing scar contraction, haemorrhage, and following aggressive fibrovascular growth (7). In the patient's LE, PCNV showed intense fibrous behaviour and, despite initiated treatment with bevacizumab, the macular region was involved and the process resulted with CNV in fovea. Consequently, the final visual acuity was affected significantly. Luckily, PCNV was discovered at initial state in the RE. Therefore, BCVA was better preserved.

According to studies, a correct pre-interventional identification of the growth site of the membrane has been shown to stratify the possibility of visual improvement after therapy. In the case described in the present study, Type I PCNV was diagnosed. It is commonly associated with AMD, as presented in this case. There are several treatment modalities including laser, photodynamic therapy with verteporfin, surgical excision, and anti-VEGF agents. Due to a small number of cases with PCNV and the lack of a large prospective placebo-controlled study, evidence-based treatment approach is not established. However, from the practical perspective, it seems logical to combine treatment modalities, as it was done in this case (2). The reported data suggest that PCNV demonstrates a very poor outcome if left untreated, with up to $25 \%$ of patients losing vision to $20 / 500$ or worse after 3 years (2). Furthermore, better outcomes are expected when treating the illness at its early stages while membranes are small and do not affect vision. This statement is in accordance with the outcome of the RE comparing to the LE. Nowadays, treatment options are focused on anti-VEGF therapy, which, theoretically, has an advantage over other management options with the purpose of maintaining the integrity of the papillomacular bundle. Early interventions could help prevent the progression of process and improve visual outcome. Singh et al concludes that anti-VEGF agents lead to significantly better visual acuity and anatomical improvement over a long-term follow-up (8).

The presented case report affirmed that early diagnosis and intervention helps maintain-visual acuity better in case of a small lesion than in large, active PCNV with oedema, hard exudates and haemorrhages. Further studies are necessary to improve evidence-based approach for PCNV.

\section{Conflict of interest: None}




\section{OD}

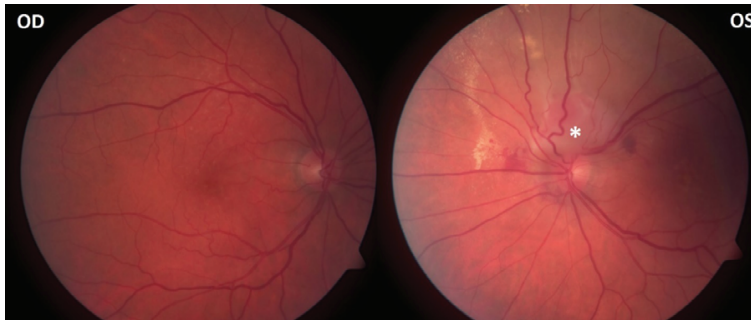

Fig. 1. A Fundus photos of the right (OD) and left (OS) eyes at the begging. In the left eye a prominent pink-coloured lesion directly above the optic nerve disc occupying 4 clock hours of the disc circumference was observed. It was surrounded by hard exudates, oedema, intraretinal and subretinal haemorrhages. In the both eyes macular drusen were seen. (* identifies the location of lesion).

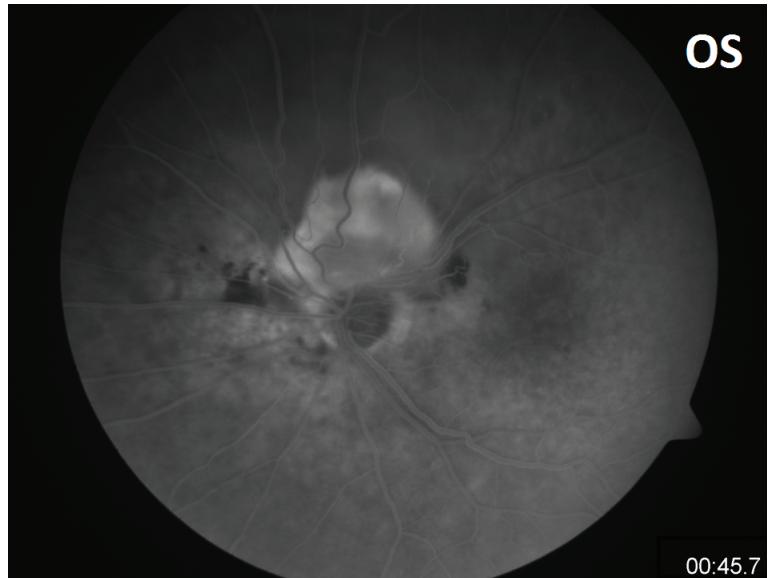

Fig. 2. Fluorescein angiography (FA) of the left (OS) eye after 45 seconds. The FA revealed hypoflourescence in an early phase followed by lacepattern hyperflourescence in an arterio-venous phase and leakage in a late phase as shown in the photo.

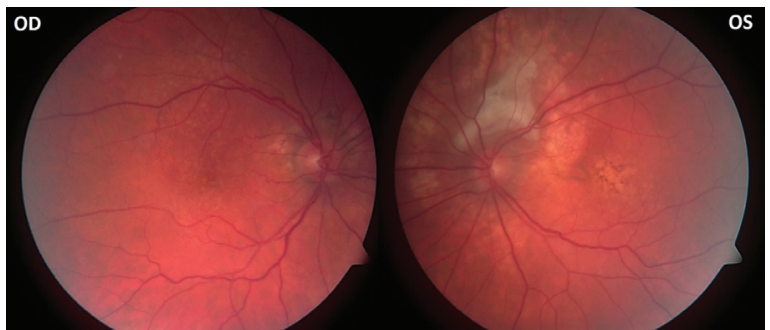

Fig. 3. A Fundus photos of the right (OD) and left (OS) eyes at the final visit. In the right eye peripapillary choroidal neovascularization was documented above the optic nerve and on the temporal-superior side. In the left eye significant subretinal fibrosis was noticed in superior/temporalsuperior part above the optic nerve disc, combined with involvement of the centre of macula and surrounded by scars of laser photocoagulation and

\section{REFERENCES}

1. Carnevali A, Cicinelli MV, Capuano V, Corvi F, Mazzaferro A, Querques L, et al. Optical Coherence Tomography Angiography: A Useful Tool for Diagnosis of Treatment-Naïve Quiescent Choroidal Neovascularization // Am J Ophthalmol, 2016; 169:189-98.

2. Jutley G, Jutley G, Tah V, Lindfield D, Menon G. Treating peripapillary choroidal neovascular membranes: a review of the evidence // Eye (Lond), $2011 ; 25(6): 675-681$

3. Browning DJ, Fraser CM. Ocular conditions associated with peripapillary subretinal neovascularization, their relative frequencies, and associated outcomes // Ophthalmology, 2005; 112:1054-1061

4. Wilde C, Patel M, Lakshmanan A, Amankwah R, Dhar-Munshi S, Amoaku W. The diagnostic accuracy of spectral-domain optical coherence tomography for neovascular age-related macular degeneration: a comparison with fundus fluorescein angiography // Eye. 2015;29:602-9.

5. Wilde C, Poostchi A, Mehta RL et al. Prevalence of peripapillary choroidal neovascular membranes (PCNV) in an elderly UK population-the Bridlington eye assessment project (BEAP): a cross-sectional study (2002-2006) // Eye (Lond), 2019;33(3):451 458.

6. Binder S. Surgical treatment of peripapillary choroidal neovascularization // Br J Ophthalmol. 2007;91:990-1.

7. Aisenbrey S, Gelisken F, Szurman P, Bartz-Schmidt KU.Surgical treatment of peripapillary choroidal neovascularisation // Br J Ophthalmol. 2007; 91(8):1027-30

8. Singh SR, Fung AT, Fraser-Bell $\mathrm{S}$ et al. Oneyear outcomes of anti-vascular endothelial growth factor therapy in peripapillary choroidal neovascularisation // Br J Ophthalmol, 2019; pii: bjophthalmol-2019-314542.

\section{Address:}

Eva Drucka, MD

Address: Riga Stradins University, Dzirciema iela 16, Riga, LV-1007, Latvia

Email: eva.drucka@gmail.com 